\title{
Farsi version of the CLEFT-Q: translation, cultural adaptation process and reliability
}

\author{
Shabnam Ajami ${ }^{1}$, Shiva Torabi², Samaneh Dehghanpour ${ }^{3 *}$ and Maryam Ajami ${ }^{4}$
}

\begin{abstract}
Background: The purpose of this study was the translation and cultural adaptation of the CLEFT-Q to Farsi and evaluating the reliability of it.

Methods: The English version of the CLEFT-Q was translated to Farsi following the guidelines set forth by the International Society for Pharmacoeconomics and Outcomes Research (ISPOR). To calculate the reliability, 50 participants filled out the Farsi version of the questionnaire twice at 2-week intervals.

Results: The difficulties during the translation and cultural adaptation process were as follows: $7.56 \%$ of items from the independent forward translations, $62.18 \%$ of items from the comparison between two forward translations, and $21 \%$ of items from the comparison between post-back translation and the original version. The internal consistency and stability of the Farsi version of the CLEFT-Q were 0.979 and 0.997 , which both were categorized as excellent.
\end{abstract}

Conclusion: The Farsi version of the CLEFT-Q is a valid and reliable tool currently available for Farsi-speaking families around the world.

Keywords: CLEFT-Q, Orofacial cleft, Health-related quality of life, Questionnaires and surveys, Result reliability

\section{Background}

One of the most common congenital abnormalities that affect many aspects of individuals' lives is cleft lip and/ or palate $(\mathrm{CL} / \mathrm{P})[1]$. Various rates of incidence have been reported in different regions of Iran [2-4]. The types of orofacial clefts have been described based on their locations and extensions [5].

$\mathrm{CL} / \mathrm{P}$ can negatively affect patients and their families in many ways like oral health-related quality of life (OHRQoL), social well-being, facial symmetry/expression, speech, and psychological problems [6, 7]. For many years, the clinician-reported outcome was the usual way to assess $\mathrm{CL} / \mathrm{P}$ treatment $[8,9]$. Patient-reported outcome (PRO) instruments provide a better understanding of the impact and effectiveness of the medical procedures

\footnotetext{
*Correspondence: Dehghanpoursamaneh@gmail.com

${ }^{3}$ Student Research Committee, School of Dentistry, Shiraz University

of Medical Sciences, Shiraz, Iran

Full list of author information is available at the end of the article
}

from patients' viewpoints for the clinicians and decisionmakers [10]. The lack of a comprehensive questionnaire specified for patients with $\mathrm{CL} / \mathrm{P}$ to evaluate the quality of their lives has been described in the literature $[11,12]$.

CLEFT-Q is a cleft-specific patient-reported outcome (PRO) instrument to assess the treatment results in children and young adults (8-29 years) with CL/P [13]. This questionnaire is a comprehensive cross-cultural tool with a CL/P focus consists of three main domains of appearance, health-related quality of life, and facial function divided into 13 scales as follows: the appearance of the face, nose, nostrils, teeth, lips, jaws, cleft scar; speech, psychological, social and school function; speech distress, and eating/drinking [13]. The content, construct and criterion validity, and reliability of the questionnaire have been evaluated in previous studies [13-16].

As a result, CLEFT-Q is a unique tool for the research field of CL/P. The goal of this study was the translation, cultural adaptation, and evaluation of the reliability of the CLEFT-Q for CL/P patients in Farsi-speaking families. 


\section{Methods}

All experimental protocols and methods in the present study were ethically approved by the Ethical Committee of Shiraz University of Medical Sciences (IR.SUMS. DENTAl.REC.1400.30). Each participant and their families signed the informed consent form to answer the questionnaire.

All methods were carried out according to the instructions of the CLEFT-Q team, based at McMaster University, Canada, which was the guideline of the International Society for Pharmacoeconomics and Outcomes Research (ISPOR) for the translation and cultural adaptation of instruments [17].

\section{Translation and cultural adaptation process}

Translation and cultural adaptation of the CLEFTQ into Farsi took place between November 2017 and November 2018. All participants of this study were patients of the cleft lip and palate clinic, Orthodontics research center, Shiraz University of Medical Sciences, who were at the clinic for their routine treatment procedures. The participants of this study were chosen conveniently.

The translation and cultural adaptation process comprised of eight steps (Fig. 1):

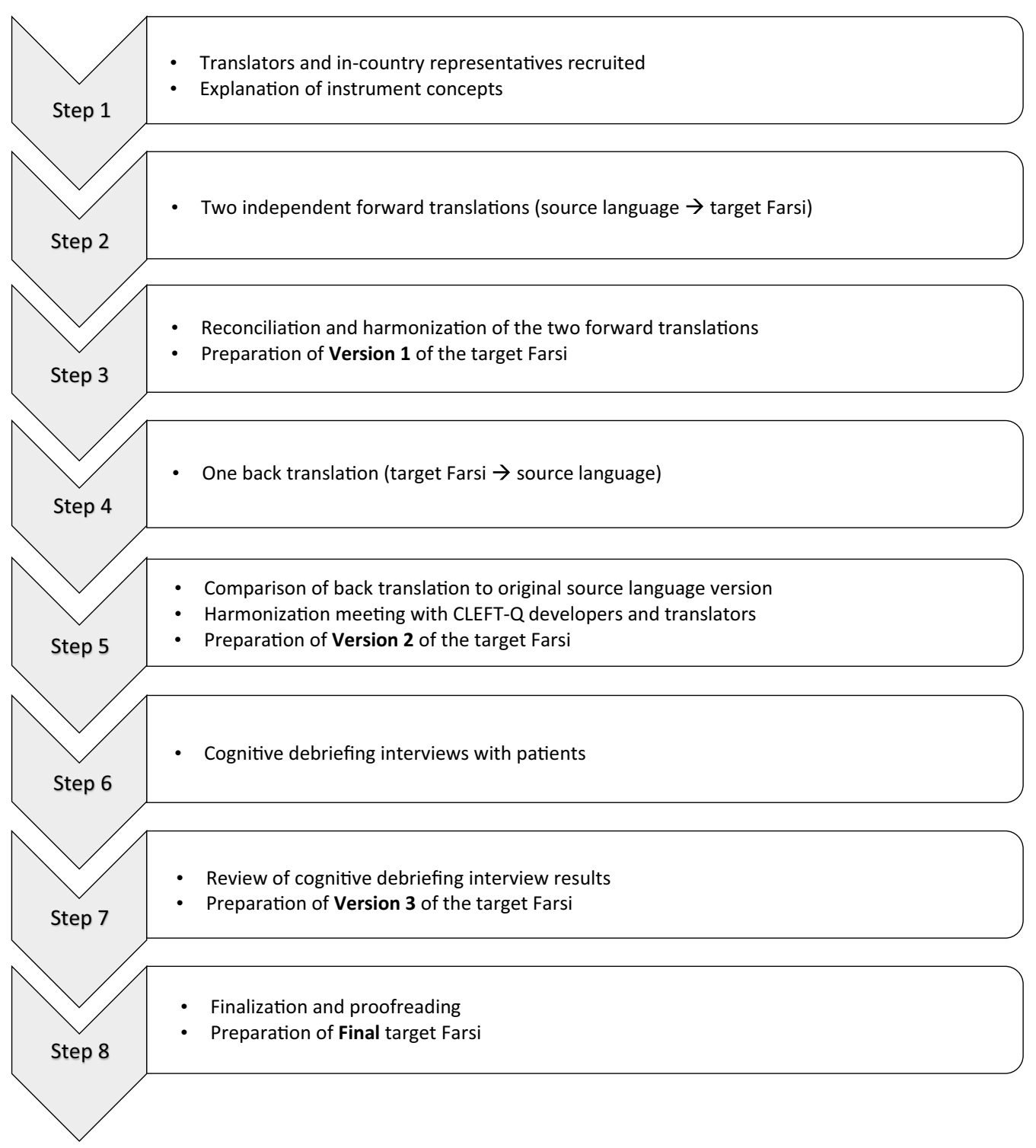

Fig. 1 Translation and cultural adaptation steps for the CLEFT-Q 


\section{Recruiting translators}

Three individuals who were fluent in English (source language) and Farsi (target language) participated in the process. Two individuals one orthodontist and one dentist who served as forward translators (English to Farsi) whose mother tongue was Farsi and fluent in English (S.D and Sh.A), and one dentist who was the back translator (Farsi to English) whose mother tongue was Farsi but has lived in an Englishspeaking country for many years (M.A). A member of the CLEFT-Q team was assigned as the project manager. The translators were instructed to provide feedback on any difficult items due to the construction, language, or cultural differences [17].

2. Independent forward translation

The forward translators separately translated the questionnaire (during 2 months period). Each translator filled out a Microsoft Excel (2016) work-sheet named "items difficult to translate".

3. Preparation of version 1 of the target language The two forward translations were compared together, and the discrepancies were identified. After the reconciliation and harmonization of the translated versions, version 1 of the Farsi questionnaire was prepared.

4. Post-back translation

Version 1 of the questionnaire was sent to the back translator for the post-back translation (Farsi to English) (during 2 months period). The back translator did not see or review the original version of the CLEFT-Q.

5. Preparation of version 2 of the target language The forward translators compared the back translation and the original version and entered the discrepancies in a Microsoft Excel (2016) work-sheet named "post-back translation comparison" (during 2 weeks period).

In this stage, all documents were sent to the project manager to solve the controversies. The project manager responded in terms of the semantic and idiomatic equivalence of the original version and the target language version (during 2 weeks period). After resolving the problems and final harmonization, version 2 of the questionnaire in the target language was prepared.
6. Cognitive debriefing interviews with patients The cognitive interviews with six patients were performed with the target patient population (mean age $=9.83$ years) to determine the quality of the translation. One of the members of the research team (Sh.T) arranged the interviews at the clinic (during 4 months period). The cognitive debriefing interviews were conducted using the "think aloud" approach (patients verbalized what the question was asking them). Each participant answered the CLEFT$Q$ verbalizing each question and what they thought it was asking $[18,19]$. Recognizing difficult items for participants was the result of this process. When any problems were found, the interviewer described the meaning to the participant, and the participant was asked to offer a substitute word/phrase to improve comprehension. The results were entered in a Microsoft Excel (2016) work-sheet named "cognitive interview reports".

7. Preparation of version 3 of the target language After the cognitive debriefing of the questionnaire according to the interview results, version 3 of the target language was prepared.

\section{Preparation of final target language version}

In this stage, all the reports and version 3 was sent to the project manager for finalization and proofreading. After careful final checks, the final version was prepared in Farsi.

\section{Reliability}

In this study, the internal consistency of the questionnaire and its scales, as well as the stability, were measured. To evaluate the internal consistency, 50 patients (mean age $=12.96$ years) with cleft lip and palate filled out the questionnaire. The test-retest method was used to calculate the stability of the CLEFT-Q in which the 50 participants answered the questionnaire for the second time at 2-week intervals. The results were interpreted as follow: "_>0.9-Excellent, __ $>0.8$-Good, _>0.7-Acceptable, _>0.6-Questionable, _>0.5-Poor, and $<0.5$-Unacceptable" [20]. Table 1 demonstrates the characteristics of the participants of this study.

\section{Statistical analysis}

Cronbach's alpha coefficient was used to calculate the internal consistency. The test-retest reliability was determined using the intraclass correlation coefficient (ICC). The results from the analyses were considered to be significant at $P<0.001$. PASW SPSS software for Windows version 18.0 (SPSS Inc., Chicago, IL, USA) was used for data analysis. 
Table 1 Characteristics of patients participating in this study (N=Number)

\begin{tabular}{lll}
\hline & $\begin{array}{l}\text { Reliability } \\
\text { assessment } \\
\mathbf{( N = 5 0 )}\end{array}$ & $\begin{array}{l}\text { Cognitive } \\
\text { debriefing } \\
\text { interviews (N=6) }\end{array}$ \\
\hline Age & $\mathrm{N}(\%)$ & $\mathrm{N}(\%)$ \\
$8-11$ & $23(46.0)$ & $6(100.0)$ \\
$12-15$ & $12(24.0)$ & $0(0.0)$ \\
$16-19$ & $11(22.0)$ & $0(0.0)$ \\
$20-23$ & $2(4.0)$ & $0(0.0)$ \\
$24-29$ & $2(4.0)$ & $0(0.0)$ \\
Gender & & $4(66.6)$ \\
Male & $31(62.0)$ & $2(33.4)$ \\
Female & $19(38.0)$ & $0(0.0)$ \\
Cleft type & & $6(100)$ \\
Cleft lip/cleft lip and alveolus & $0(0.0)$ & $0(0.0)$ \\
Cleft lip and palate & $50(100.0)$ & \\
Cleft palate only & $0(0.0)$ &
\end{tabular}

\section{Results}

\section{Translation process}

After the independent forward translation, the translators described that 9 items out of a total of 119 items $(7.56 \%)$ were difficult to translate. For instance, items such as "How much do you like the color of your cleft lip scar?" and "I feel upset when I am not understood" were difficult to translate, as the use of the verb "like" with the word "scar", and "I am not understood" are phrases not commonly used in Farsi. The comparison of the two forward translations revealed the inconsistency of 74 items $(62.18 \%)$ which were related to the wording or phrasing of the items.

The comparison between the original version and the back-translation version showed that in 25 items of the questionnaire $(21 \%)$, semantic, idiomatic, or conceptual equivalence was not achieved. The Additional file 1: Table S1 shows some of these items in more detail (see Additional file 1: Table S1). None of the participants in cognitive debriefing interviews reported any difficulties.

\section{Reliability}

Table 2 shows the results of the internal consistency of the Farsi version of the CLEFT-Q which was 0.979 overall. The results were categorized as excellent on each scale except for the "drinking and eating" scale. The outcomes of test-retest reliability are indicated in Table 3. The overall stability was 0.997 . The categorization of the stability results was similar to the results of internal consistency in all scales.
Table 2 Results of the internal consistency of the Farsi version of the CLEFT-Q

\begin{tabular}{|c|c|c|c|}
\hline Scale of the CLEFT-Q & Cronbach's alpha & Interpretation & $\begin{array}{l}\text { Number } \\
\text { of items }\end{array}$ \\
\hline Appearance of the face & 0.958 & Excellent & 9 \\
\hline $\begin{array}{l}\text { Appearance of the } \\
\text { nose }\end{array}$ & 0.979 & Excellent & 12 \\
\hline $\begin{array}{l}\text { Appearance of the } \\
\text { nostrils }\end{array}$ & 0.981 & Excellent & 6 \\
\hline $\begin{array}{l}\text { Appearance of the } \\
\text { teeth }\end{array}$ & 0.967 & Excellent & 8 \\
\hline Appearance of the lips & 0.966 & Excellent & 9 \\
\hline $\begin{array}{l}\text { Appearance of the } \\
\text { lip scar }\end{array}$ & 0.983 & Excellent & 7 \\
\hline Appearance of the jaws & 0.962 & Excellent & 7 \\
\hline Speech function & 0.955 & Excellent & 12 \\
\hline Speech distress & 0.932 & Excellent & 10 \\
\hline Psychological function & 0.981 & Excellent & 10 \\
\hline School function & 0.927 & Excellent & 10 \\
\hline Social function & 0.918 & Excellent & 10 \\
\hline Drinking and eating & 0.798 & Acceptable & 9 \\
\hline Total & 0.979 & Excellent & 119 \\
\hline
\end{tabular}

\section{Discussion}

The translation and cultural adaptation of the CLEFT-Q was done according to best-practice guidelines [17] and in a standard method. All scales of the questionnaire were found to be comprehensible and suitable by the participants. The Farsi version of the CLEFT-Q also showed an excellent rate of reliability.

Table 3 Results of the test-retest stability of the Farsi version of the CLEFT-Q

\begin{tabular}{lllll}
\hline Scale of the CLEFT-Q & ICC & P value & Interpretation & $\begin{array}{l}\text { Number } \\
\text { of items }\end{array}$ \\
\hline Appearance of the face & 0.989 & $\mathrm{~S}$ & Excellent & 9 \\
Appearance of the nose & 0.993 & $\mathrm{~S}$ & Excellent & 12 \\
Appearance of the nostrils & 0.943 & $\mathrm{~S}$ & Excellent & 6 \\
Appearance of the teeth & 0.990 & $\mathrm{~S}$ & Excellent & 8 \\
Appearance of the lips & 0.990 & $\mathrm{~S}$ & Excellent & 9 \\
Appearance of the lip scar & 0.954 & $\mathrm{~S}$ & Excellent & 7 \\
Appearance of the jaws & 0.995 & $\mathrm{~S}$ & Excellent & 7 \\
Speech function & 0.996 & $\mathrm{~S}$ & Excellent & 12 \\
Speech distress & 0.991 & $\mathrm{~S}$ & Excellent & 10 \\
Psychological function & 0.995 & $\mathrm{~S}$ & Excellent & 10 \\
School function & 0.990 & $\mathrm{~S}$ & Excellent & 10 \\
Social function & 0.992 & $\mathrm{~S}$ & Excellent & 10 \\
Drinking and eating & 0.757 & $\mathrm{~S}$ & Acceptable & 9 \\
Total & 0.997 & $\mathrm{~S}$ & Excellent & 119 \\
\hline
\end{tabular}

$S$, significant $(P<0.001)$, ICC, intraclass correlation coefficient 
The CLEFT-Q was cross-culturally developed for the international application of patients with different age ranges who were born with $\mathrm{CL} / \mathrm{P}[13,15]$. The basis of the scales in the CLEFT-Q was a conceptual framework designed by in-depth interviews with 136 patients with clefts of any age across six countries [21]. Furthermore, collected data from 2434 participants, aged 8 to 29 years from 12 countries, was used to establish norm values of the CLEFT-Q [15]. Therefore, CLEFT-Q is one of the most efficient available PRO instruments specified for patients born with CL/P. As a result, developing a Farsi version of the CLEFT-Q seemed to be essential.

The ultimate aim of the translation process was to achieve a precise conceptual translation rather than a literal one, which called for a scientific methodology to achieve complete equality of the questions, instructions, and response options. The ISPOR provided a standard method to accomplish the goals of the translation and cultural adaptation process [17]. Cognitive debriefing interviews were one of the most vital steps of the translation process. The participation of a few patients certified the ease of understanding and application of the Farsi version of the CLEFT-Q [22].

No significant challenge emerged during the translation and cultural adaptation process. Constant consultation and coordination between the translators and the project manager allowed the team to follow the process accurately and scientifically. The team members anticipated that the cognitive interviews would lead to face some problems with children, especially the ones between ages 8 to 10 , due to their lesser ability to read texts. Surprisingly, even the slow-readers could comprehend the items of the questionnaire easily. Several studies reported that children as young as 8 years age are capable of providing self-reported outcomes [23-25]. However, inadequate formal education of some of the children from the deprived regions and the length of the interview arose some challenges during the process.

The field-test version of the CLEFT-Q consisted of 154 items with 13 scales was translated to Colombian, Chilean, Spanish, Arabic, Dutch, Hindi, Swedish, and Turkish [22, 26]. After forward translation to Farsi, $7.54 \%$ of the items, were found difficult to translate. Only Arabic and Swedish forward translators reported a higher rate of difficulties comparing to Farsi, and the rest of the translators faced fewer problems [22, 26]. The inconsistency between the two forward translations was $62.18 \%$. The rate of inconsistency in Turkish and Colombian translations was above the Farsi translation, while other languages translators stated lower rates $[22,26]$. This relatively high rate of inconsistency was the result of slightly different approaches of the forward translators, which was raised at the consensus meeting. One of the translators was trying to keep the translation literally as possible, while the other one was to achieve the conceptual translation. Comparing the original version and back-translated version of the CLEFT-Q resulted in finding that $21 \%$ of items didn't have semantic, idiomatic, or conceptual equivalence. This rate was more than all other languages except for Turkish $[22,26]$. These various rates are the result of the significant grammatical differences between the languages and their diverse origins. None of the Farsispeaking participants in cognitive debriefing interviews stated any problem, while some difficulties during the interviews in other languages were reported $[22,26]$. This may be relevant to the fact that the Farsi version of the CLEFT-Q was translated from the final English version, while the other translations were done from the field-test version of the CLEFT-Q.

The evaluation of the reliability of the Farsi version of the CLEFT-Q demonstrated excellent internal consistency and stability. It might seem that the relatively high results of reliability were because of the multiple numbers of the questionnaire items [27]. But the outcome didn't decrease significantly in each scale with fewer questions. The CLEFT-Q scales can operate independently (meaning only relevant scales to each specific individual can be used) $[13,28]$. Therefore, the reliability of each scale, as well as the whole questionnaire, was assessed. The internal consistency and the stability of all scales were categorized as excellent except for the "drinking and eating" scale. It might be because the participants suffered from varying degrees of food regurgitation as one of the complications of palatal repair surgery.

Following one of the best available guidelines for the translation and cultural adaptation, adequate size of the sample to assess the reliability, and recruiting participants from various socio-economics status and regional cultures were the strengths of this study. A potential limitation is that the back-translator mother tongue was not English, which differed from the ISPOR recommendations. Nonetheless, it seems improbable that this slight deviation from the ISPOR guideline has influenced the quality of the translation. The fact that participants of cognitive debriefing interviews were not from a wide age range and all different kinds of $\mathrm{CL} / \mathrm{P}$ is another possible limitation. However, children were more likely to report difficulties comprehending the items of the questionnaire than the older individuals. Besides, patients with cleft lip and palate usually struggle with problems that other categories of individuals with CL/P would face them too. The mentioned reasons would ensure that the participants were a representative sample. 


\section{Conclusion}

The Farsi version of the CLEFT-Q is a valid and reliable tool currently available for Farsi-speaking families around the world.

\section{Abbreviations}

CL/P: Cleft lip and/or palate; PRO: Patient-reported outcome; ISPOR: International Society for Pharmacoeconomics and Outcomes Research; ICC: Intraclass correlation coefficient.

\section{Supplementary Information}

The online version contains supplementary material available at https://doi. org/10.1186/s12903-021-01957-7.

Additional file 1. Table S1. Examples of Discrepancies between the Original English Version and Back-translated English Version.

\section{Acknowledgements}

We would like to sincerely thank Professor Elena Tsangaris who helped us through the translation and cultural adaptation process as our project manager. We also would like to thank McMaster University and the CLEFT-Q team, especially Professor Anne F. Klassen, for their excellent cooperation and kind assistance during the process.

\section{Authors' contributions}

SA and SD served as forward translators in the process of translation and cultural adaptation of CLEFT-Q. They also contributed in analyzing and interpreting the patients' data, and writing the manuscript. ST conducted all the interviews with the patients throughout the study. MA served as the backtranslator in the process of translation and cultural adaptation of CLEFT-Q. All authors read and approved the final manuscript.

\section{Funding}

This study was sponsored by Shiraz University of Medical Sciences under code (18396).

\section{Availability of data and materials}

The data and materials that support the findings of this study are available from McMaster University, Canada, but restrictions apply to the availability of these data and materials, which were used under license for the current study, and so are not publicly available. Data are however available from the authors upon reasonable request and with permission of McMaster University, Canada.

\section{Declarations}

Ethics approval and consent to participate

All experimental protocols and methods in the present study was ethically approved by the Ethical Committee of Shiraz University of Medical Sciences (IR.SUMS.DENTAI.REC.1400.30). Each participant and their families signed the informed consent form to answer the questionnaire.

\section{Consent for publication}

Not applicable.

\section{Competing interests}

The authors declare no competing interests.

\section{Author details}

${ }^{1}$ Orthodontics Research Center, School of Dentistry, Shiraz University of Medical Sciences, Shiraz, Iran. ${ }^{2}$ Department of Orthodontics, School of Dentistry, Shiraz University of Medical Sciences, Shiraz, Iran. ${ }^{3}$ Student Research Committee, School of Dentistry, Shiraz University of Medical Sciences, Shiraz, Iran. ${ }^{4}$ San Francisco, USA.
Received: 9 July 2021 Accepted: 9 November 2021

Published online: 19 November 2021

\section{References}

1. Silva M, Balderrama IF, Wobeto AP, Werneck Rl, Azevedo-Alanis LR. The impact of nonsyndromic cleft lip with or without cleft palate on oral health-related quality of life. J Appl Oral Sci. 2018;26:e20170145.

2. Rajabian $\mathrm{MH}$, Aghaei S. Cleft lip and palate in southwestern Iran: an epidemiologic study of live births. Ann Saudi Med. 2005:25(5):385-8.

3. Mirfazeli A, Kaviany N, Hosseinpour KR, Golalipour MJ. Incidence of cleft lip and palate in Gorgan-Northern Iran: an epidemiological study. Oman Med J. 2012;27(6):461-4.

4. Kianifar $\mathrm{H}$, Hasanzadeh $\mathrm{N}$, Jahanbin A, Ezzati A, Kianifar H. Cleft lip and palate: a 30-year epidemiologic study in North-East of Iran. Iran J Otorhinolaryngol. 2015;27(78):35-41.

5. Mossey PA, Little J, Munger RG, Dixon MJ, Shaw WC. Cleft lip and palate. Lancet. 2009;374(9703):1773-85.

6. Montes ABM, Oliveira TM, Gavião MBD, Barbosa TS. Orofacial functions and quality of life in children with unilateral cleft lip and palate. Braz Oral Res. 2019:33:e0061.

7. De Cuyper E, Dochy F, De Leenheer E, Van Hoecke H. The impact of cleft lip and/or palate on parental quality of life: a pilot study. Int J Pediatr Otorhinolaryngol. 2019;126:109598.

8. Long RE Jr, Hathaway R, Daskalogiannakis J, Mercado A, Russell K, Cohen $M$, et al. The Americleft study: an inter-center study of treatment outcomes for patients with unilateral cleft lip and palate part 1. Principles and study design. Cleft Palate Craniofac J. 2011;48(3):239-43.

9. Heliövaara A, Küseler A, Skaare P, Shaw W, Mølsted K, Karsten A, et al. Scandcleft randomised trials of primary surgery for unilateral cleft lip and palate: 6. Dental arch relationships in 5 year-olds. J Plast Surg Hand Surg. 2017:51(1):52-7.

10. Acquadro C, Berzon R, Dubois D, Leidy NK, Marquis P, Revicki D, et al. Incorporating the patient's perspective into drug development and communication: an ad hoc task force report of the Patient-Reported Outcomes (PRO) Harmonization Group meeting at the Food and Drug Administration, February 16, 2001. Value Health. 2003;6(5):522-31.

11. Eckstein DA, Wu RL, Akinbiyi T, Silver L, Taub PJ. Measuring quality of life in cleft lip and palate patients: currently available patient-reported outcomes measures. Plast Reconstr Surg. 2011;128(5):518e-e526.

12. Klassen AF, Tsangaris E, Forrest CR, Wong Riff KWY, Pusic AL, Cano SJ, et al. Quality of life of children treated for cleft lip and/or palate: a systematic review. J Plast Reconstr Aesthet Surg. 2012;65(5):547-57.

13. Tsangaris E, Wong Riff KWY, Goodacre TEE, Forrest CR, Dreise M, Sykes J, et al. Establishing content validity of the CLEFT-Q: a new patient-reported outcome instrument for cleft lip/palate. Plast Reconstr Surg Glob Open. 2017;5(4):e1305.

14. Wong Riff KWY, Tsangaris E, Goodacre TEE, Forrest CR, Pusic AL, Cano SJ, et al. International multiphase mixed methods study protocol to develop a cross-cultural patient-reported outcome instrument for children and young adults with cleft lip and/or palate (CLEFT-Q). BMJ Open. 2017:7(1):e015467.

15. Klassen AF, Wong Riff KWY, Longmire NM, Albert A, Allen GC, Aydin MA, et al. Psychometric findings and normative values for the CLEFT-Q based on 2434 children and young adult patients with cleft lip and/or palate from 12 countries. CMAJ. 2018:190(15):e455-62.

16. Harrison CJ, Rae C, Tsangaris E, Wong Riff KWY, Swan MC, Goodacre TEE, et al. Further construct validation of the CLEFT-Q: ability to detect differences in outcome for four cleft-specific surgeries. J Plast Reconstr Aesthet Surg JPRAS. 2019;72(12):2049-55.

17. Wild D, Grove A, Martin M, Eremenco S, McElroy S, Verjee-Lorenz A, et al. Principles of good practice for the translation and cultural adaptation process for patient-reported outcomes (PRO) measures: report of the ISPOR task force for translation and cultural adaptation. Value Health. 2005:8(2):94-104

18. Willis GB. Cognitive interviewing as a tool for improving the informed consent process. J Empir Res Hum Res Ethics. 2006;1(1):9-24.

19. Willis $G B$. Analysis of the cognitive interview in questionnaire design: understanding qualitative research. Toronto: Oxford University Press; 2015. 
20. Gliem J, Gliem R. Calculating, interpreting, and reporting Cronbach's alpha reliability coefficient for likert-type scales 2003. In: Midwest research to practice conference in adult, continuing, and community education; 2003. p. 82-8.

21. Wong Riff KWY, Tsangaris E, Goodacre TEE, Forrest CR, Lawson J, Pusic AL, et al. What matters to patients with cleft lip and/or palate: an international qualitative study informing the development of the CLEFT-Q. Cleft Palate Craniofac J. 2018;55:442-50.

22. Tsangaris E, Wong Riff KWY, Vargas F, Aguilera MP, Alarcón MM, Cazalla AA, et al. Translation and cultural adaptation of the CLEFT-Q for use in Colombia, Chile, and Spain. Health Qual Life Outcomes. 2017;15(1):228.

23. Varni JW, Limbers CA, Burwinkle TM. How young can children reliably and validly self-report their health-related quality of life? An analysis of 8,591 children across age subgroups with the PedsQL 40 Generic Core Scales. Health Qual Life Outcomes. 2007;5:1.

24. Bevans KB, Riley AW, Moon J, Forrest CB. Conceptual and methodological advances in child-reported outcomes measurement. Expert Rev Pharmacoecon Outcomes Res. 2010;10(4):385-96.
25. Arbuckle R, Abetz-Webb L."Not just little adults": qualitative methods to support the development of pediatric patient-reported outcomes. Patient. 2013;6(3):143-59.

26. Tsangaris E, Wong Riff KWY, Dreise M, Stiernman M, Kaur MN, Piplani $B$, et al. Translation and cultural adaptation of the CLEFT-Q into Arabic, Dutch, Hindi, Swedish, and Turkish. Eur J Plast Surg. 2018;41 (6):643-52.

27. Kimberlin CL, Winterstein AG. Validity and reliability of measurement instruments used in research. Am J Health Syst Pharm. 2008:65(23):2276-84

28. Harrison CJ, Geerards D, Ottenhof MJ, Klassen AF, Wong Riff KWY, Swan MC, et al. Computerised adaptive testing accurately predicts CLEFT-Q scores by selecting fewer, more patient-focused questions. J Plast Reconstr Aesthet Surg. 2019;72(11):1819-24.

\section{Publisher's Note}

Springer Nature remains neutral with regard to jurisdictional claims in published maps and institutional affiliations.
Ready to submit your research? Choose BMC and benefit from:

- fast, convenient online submission

- thorough peer review by experienced researchers in your field

- rapid publication on acceptance

- support for research data, including large and complex data types

- gold Open Access which fosters wider collaboration and increased citations

- maximum visibility for your research: over $100 \mathrm{M}$ website views per year

At BMC, research is always in progress.

Learn more biomedcentral.com/submissions 